\title{
NACHTRAG ZU HEFT 3-2015
}

Im Artikel „Die Linie der Infekte und das Metagenom“ in Heft 3-2015 fehlt auf Seite 27 die zum Hinweis auf Tabelle 1 passende zugehörige Tabelle. Damit wird Tabelle1 im Artikel Heft 3-2015 auf Seite 28 zu Tabelle 2.
Beide Tabellen sind hiermit nachgereicht und korrigiert. Wir bitten um Nachsicht. Wer gern den korrigierten Artikel haben möchte wende sich bitte an klowersa@gmail. com. Er wird Ihnen zugesandt.

Tabelle 1: Blütentherapie spezifischer Erreger

\begin{tabular}{|l|l|l|}
\hline Erreger & Blütenessenz & Einnahme \\
\hline Anaplasma/Ehrlichia & Star of Bethlehem (ALE) & Einnahme ca. 6 Mt. \\
\hline Ascaris (Spulwurm) & Southern Cross (ALE) & Einnahme ca. 6 Mt. \\
\hline Bartonella & Rabbitbrush (FES) & Einnahme ca. 6 Mt. \\
\hline Borrelia & White Spider Orchid (ALE) und Ursinia (ALE) & Einnahme ca. 18 Mt. \\
\hline Chlamydia & Freshwater Mangrove (ABFE) & Einnahme ca. 6 Mt. \\
\hline Coxiella b. & One Sided Bottlebrush (ALE) & Einnahme ca. 6 Mt. \\
\hline EB-Virus & She Oak (ABFE) & Einnahme ca. 6 Mt. \\
\hline Herpes Zoster & Macrozamia (ALE) & bis zur Symptomfreiheit \\
\hline Influenza: Saisonale und Schweinegrippe & Kangaroo Paw (ABFE) & bis zur Symptomfreiheit \\
\hline Giardia lamblia & Brachycome (ALE) & Einnahme ca.6 Mt. \\
\hline Mycoplasmatacea & Sagebrush (FES) & Einnahme ca. 6 Mt. \\
\hline Rickettsia & Philoteca (ABFE) & Einnahme ca. 6 Mt. \\
\hline Toxoplasma & Little Flannel Flower (ABFE) & Einnahme ca. 6 Mt. \\
\hline ALE: Australische Livingessenz, ABFE: Australische Buschblüten, FES: Kalifornische Blüten & \\
\hline & & \\
\hline
\end{tabular}

\section{Tabelle 2: Behandlungsschema}

\section{Zuerst Behandlung Oszillation, Inversion}

Dominant Auflage Frequenz B, Suche nach aktiven Punkten mit Clematis Ampulle oder Frequenz $7712 \mathrm{~Hz}$

Aktive Punkte ( 1 bis 4 Punkte) dauerstimulieren: Repetitiv ASP oder besser ab dem 2. Mal Templax, evtl. vorgängig zwei Monate White Spider Orchid Energetische Stabilisierung: Stimulation von Leber, ACTH und Lexotanil ${ }^{\circledR}$ Pkt. mit Dauernadeln u.a.

Drainage bei der Borreliose!

Therapiekontrolle (Linie der Infekte stumm?)

Nachkontrollen!

\section{Augenärztin mit Schwerpunkt TCM}

sucht Kollegen/ in anderer Fachrichtung

zur Gründung einer gemeinsamen

TCM Praxis in Zürich oder würde gerne in einer schon bestehenden Praxisgemeinschaft mitarbeiten.

Kontakt: Erika@diewegscheiders.de 